\title{
The Role of Mindfulness and Self Compassion toward Depression among Pregnant Women
}

\author{
Endang Fourianalistyawati, Aisyah Uswatunnisa, Alfira Chairunnisa
}

Faculty of Psychology, Universitas YARSI, Indonesia

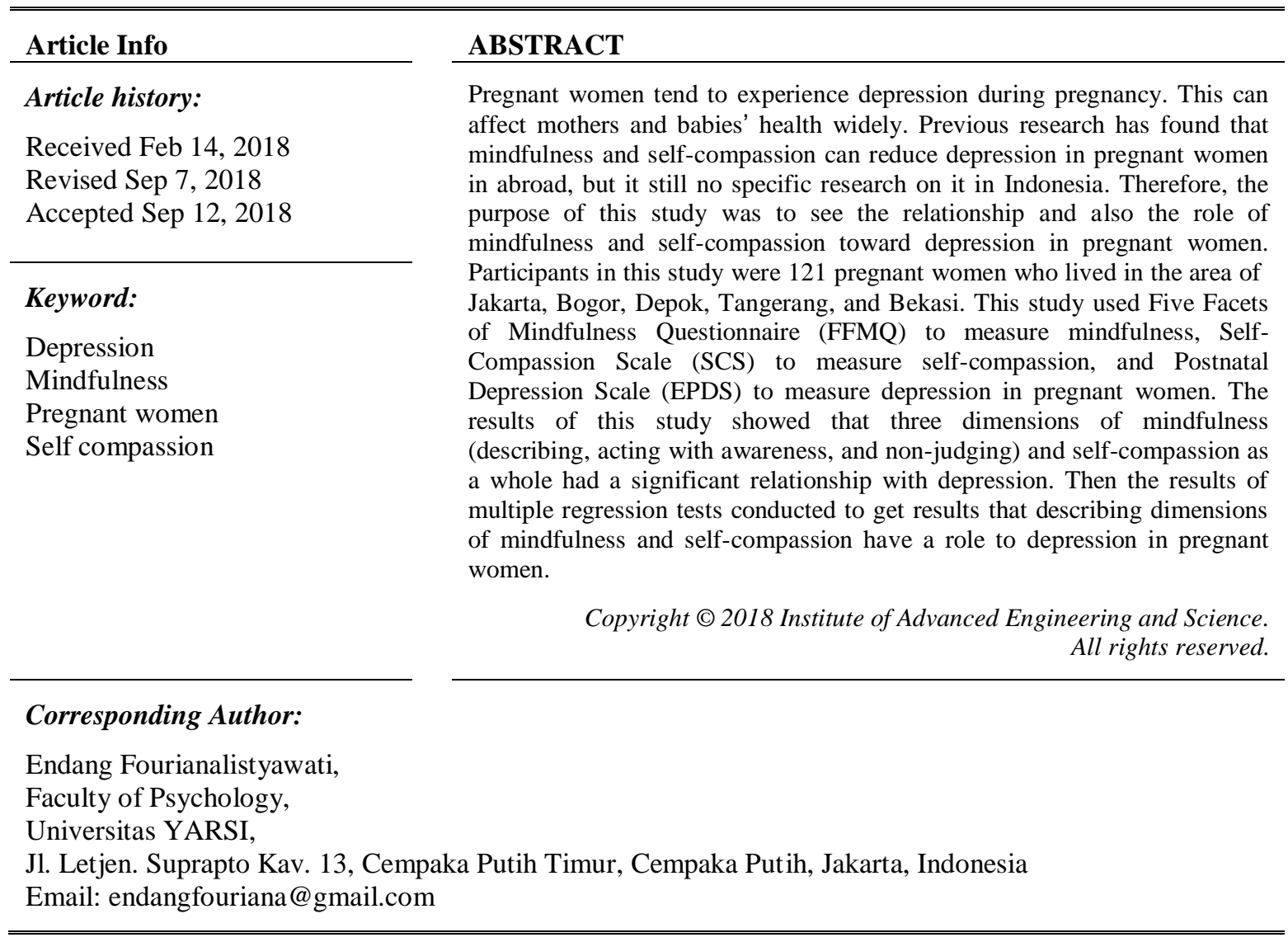

\section{INTRODUCTION}

Pregnant women have some inevitable changes in their life, such as hormonal changes and body structure changes [1]. Pregnant women may be afraid of childbirth because the pregnancy is their first pregnancy, or the mother has had a negative experience of labor before. These experiences can lead to depression in pregnant women [2]. Depression in pregnant women can also occur because of these following factors: previous depression experience, young age pregnancy, low social economic status, previous violence experience or during pregnancy, disharmony relationship with spouse, living alone without spouse, previous miscarriage experience, unwanted pregnancies, having many children, and lack of social support [3].

The prevalence of depression symptoms in pregnant women around the world was estimated at $6.5 \%$ to $12.9 \%$ [4]. It was estimated that $7.4 \%$ of pregnant women experience it during the first trimester, $12.8 \%$ during the second trimester, and $12 \%$ during the last trimester [5]. Meanwhile, there are no definite numbers related to the prevalence of depression experienced by pregnant women until now in Indonesia. Nevertheless, from research conducted by Roomruangwong and Epperson [6], it was found that the prevalence of depression in pregnant women in Asia was slightly higher than in other western countries. There are several unique factors that cause depression of pregnant women in Asia, for instance: conflict with in-laws (especially mother-in-law), dissatisfaction of children' sex (mostly girls), unwed pregnancy, poverty or financial difficulties, lack of social support, marital dissatisfaction or marital conflicts [6]. The previous 
study showed that $50 \%$ of the high risk participants of pregnant women experienced depression symptoms [7]. Previous study conducted in Indonesia related to depression in pregnant women by Handayani and Fourianalistyawati [8] also found result that $20 \%$ of participants of pregnant women experienced symptoms of depression.

Depression may have a negative impact on pregnant women. Pregnant women whose depression was not treated early could result in difficult adjustment between mother and child during perinatal and postpartum periods [2]. Moreover, other studies have also found that depression experienced during pregnancy has an impact on childbirth, such as complications during childbirth, emergence of symptoms during pregnancy, premature birth and use of pain relief during childbirth [10].

Depression experienced during pregnancy may persist until post birth if it is not treated well since pregnancy [12]. It can lead to negative impact of the fetus. Symptoms of depression and anxiety experienced during pregnancy can also lead to the risk of premature births of fetus as well as low birth weight [11]. Previous study conducted by Deave, Heron, Evans, and Emond [9] found that depression during pregnancy can cause development delay of children. Depression that continues post birth process can disrupt the process of adaptation of mother and child, and negatively impact on attachment and infant care of mother to child [12].

Pregnant women who experienced depression can be help primarily using drugs. However, they tend to hesitate to using drugs during pregnancy due to fear of stigma and side effects of it for the fetus [13]. Therefore, other interventions to reduce depression on pregnant women with fewer side effects on both pregnant mother and the fetus are needed. One of the factors that can help reducing depression levels of pregnant women is mindfulness.

Mindfulness comes from the psychology of Buddhism. Mindfulness approach concerns on quality of attention and awareness of a person, which can be developed and trained through meditation. The definition of mindfulness is the awareness that arises by focusing attention at this moment by not judging the perceived experience [14]. In mindfulness, a person directs his/her attention to his/her present mind and world; and his/her current experiences such as feelings and body sensations, external stimuli such as sight, sound and smell [15]. Mindfulness-based interventions have been developed to overcome depression of pregnant women. From previous studies, concluded that mindfulness can decrease the symptoms of depression, anxiety, or psychological distress experienced by pregnant women $(16,17,18,19,2]$.

According to Baer, Smith, Hopkins, Krietemeyer, and Toney [20], mindfulness is divided into five dimensions: observing, describing, acting with awareness, none judging of inner experience and non reactivity to inner experience. The observing dimension discusses on how person can be aware of the external and internal experiences received by himself, such as sensation, cognition, emotion, sight, sound and smell. The dimension of describing is about the way people label external experience with words. The dimension of acting with awareness is about the ability of a person to perform an activity with focus and awareness. The nonjudging of inner experience dimension is the situation when a person does not give an assessment of what he feels or thinks about. The dimension of nonreactivity to inner experience is condition which a person receives the thoughts and feelings just the way it is, and he is able to let it go without being dissolved and carried away by that feeling or thought.

In addition to mindfulness, self-compassion is also found to have a relationship with depression on pregnant women [21]. Depressed pregnant women are found to have low self-compassion levels [21]. Self-compassion is the way a person opens his self to the suffering that he experiences, cares about himself, understands himself, does not judge his inadequacies and failures, and realizes that the experience is also a common experience for everyone [22]. A person who has self-compassion has the three basic components in his self. First, a person is able to expand the kindness and understanding of himself, rather than giving criticism and self-assessment. The second, a person is able to understand that an experience he experiences can happen to every individual, rather than feeling isolated. The third is that individual is able not to get carried away of his sad thoughts or feelings, and remains aware of the sad thoughts and feelings [23]. Selfcompassion helps people to face negative emotions in awareness with kindness, understanding and a sense of shared humanity. Self-compassion requires people to not over-identification and harshly judgmental of painful or distressing feeling, but seen it clearly as one of the common human experience [23]. People will get a thorough understanding of their situations and adopting proactive behaviors to change the situations in an effective way and maintaining their well-being. In previous research, it was found that self-compassion could improve quality of life and have a role in predicting mental health on pregnant women [24]. When a person has a good self-compassion, the person will feel more attached and secure. Self-compassion can also help mother and fetus to have better attachment [24].

A study conducted by Baer, Lykins, and Peters [15] found that mindfulness and self-compassion related to psychological well-being. However, there is still no research in Indonesia related to mindfulness, self-compassion and depression on pregnant women. Before developing mindfulness-based intervention, 
we need to know the relationship and role of mindfulness and self-compassion toward depression of pregnant women in Indonesia. Therefore, we were interested to examine mindfulness, self-compassion and depression on pregnant women in Indonesia. This research is expected to be the basic evidence for the development of alternative approaches for pregnant women using mindfulness-based interventions in Indonesia.

\section{RESEARCH METHOD}

Participants in this study were 121 pregnant women with specific criteria above 18 years and live in the area of Jakarta, Bogor, Depok, Tangerang, and Bekasi. The sample selection technique used purposive sampling. Participants in this study were obtained through online questionnaire. The statistical calculation in this research was done using JASP 0.8.2.0 software. Authorization of sample collection was obtained from Ethic Committee of the Universitas YARSI and Ethic Committee of the Faculty of Psychology of the Universitas YARSI. Samples were obtained by personal link and online. Subjects were given informed consent before fill the questionnaire.

The Five Facets of Mindfulness Questionnaire (FFMQ) [20] that have been adapted into Indonesian was used to collect data. FFMQ consists of 39 items and divided into 5 dimensions namely: observing, describing, acting with awareness, non-judging of inner experience, and non-reactivity to inner experience. This measure used Likert scale which was ranged from 1 (never) to 5 (always). In this study, a preliminary data was conducted to 30 pregnant women to see the validity and reliability on a scale that has been adapted into the Indonesian language.

Furthermore, Self-Compassion Scale to gather data (SCS) [23] which has been adapted into Indonesian was also used. Result of premilinary data showed that SCS was reliable (0.92). The internal consistency of the scale was $(0.385-0.719)$. The scale consists of 26 items and has 6 aspects consists of selfkindness, self-judgment, common humanity, isolation, mindfulness, and over-identified. This scale can measure the level of one's self-compassion in every aspect or as a whole. This study used self-compassion score as a whole. The measurement used Likert scale from 1 (almost never) up to 5 (almost always).

Depression in this study was measured using Edinburgh Postnatal Depression Scale (EPDS) [25] that has been adapted into Indonesian. Result of premilinary data showed that EPDS was reliable (.834). The internal consistency of the scale was (0.239-0.734). EPDS is a depression measuring tool that has been used to measure depression in pregnant women. This measuring instrument consists of 10 items. The depression cut-off score used in this study was 12 . Hence, if a person has a total depression score above 12 , it means he/she has the possibility of suffering from depression and requiring further clinical diagnosis.

\section{RESULTS AND ANALYSIS}

\subsection{Demographic Results}

Table 1 is about demographic data. It was found that $57 \%$ of the participants in the study were $26-32$ years old. The majority of participants lived in Jakarta were $71.1 \%$. Moreover, $34.7 \%$ have a undergraduate education background, $96.7 \%$ of participants were in their first marriage, 52.9\% subjects were on the first pregnancy, $43.8 \%$ of participants had entered the third trimester, and $93.4 \%$ of participants in this study were never had a miscarriage previously. There were $32.2 \%$ of participants in the study were depressed and $67.8 \%$ were not depressed.

\subsection{Correlation Test}

The first hypothesis in this study was mindfulness and self-compassion have a relationship with depression. Pearson correlation was used to know the correlation between the three variables. Table 2 shows a significant correlation between self-compassion and depression. However, there were only three dimensions associated with depression: describing, acting with awareness, and non-judging. Therefore, the observing and non-reactivity dimensions could not be included in the regression analysis.

\subsection{Multiple Regression Test}

The second hypothesis in this study was mindfulness and self-compassion can predict depression significantly. There were two independent variables in this study so we need to do multiple regression analysis. Based on Table 3, it showed that the dimensions of describing, acting with awareness, non-judging together with self-compassion have a significant role in predicting depression $(\mathrm{p}=0.000)$. Mindfulness and self-compassion had a significant role that was equal to $42.4 \%$, meanwhile $57.6 \%$ influenced by other factors.

Table 4 shows about B-Values \& Sig. The regression test results also showed that the dimensions of mindfulness that contribute significantly in predicting depression was describing dimensions $(\beta=-0.203$, 
$\mathrm{p}=0.009)$, while the acting with awareness and non-judging dimensions could not predict significantly. Selfcompassion as a whole was also found to have contributed significantly in predicting depression $(\beta=-0.340$, $\mathrm{p}=0.000)$.

Table 1. Demographic Data

\begin{tabular}{lcc}
\hline & Variable & Percentage \\
\hline Age & $19-25$ years old & $28.1 \%$ \\
& $26-32$ years old & $57 \%$ \\
& $33-39$ years old & $13.2 \%$ \\
Domicile & $40-46$ years old & $1.7 \%$ \\
& Jakarta & $71.1 \%$ \\
& Bogor & $4.1 \%$ \\
& Depok & $7.4 \%$ \\
Tangerang & $2.5 \%$ \\
Education & Bekasi & $14.9 \%$ \\
& Elementary School & $2.5 \%$ \\
& Junior High School & $7.4 \%$ \\
& Senior High School & $28.9 \%$ \\
& Diploma 1 & $2.5 \%$ \\
& Diploma 2 & $19 \%$ \\
Marital Status & S1 & $34.7 \%$ \\
Birth Status & S2 & $5 \%$ \\
& Married (First Married) & $96.7 \%$ \\
Pregnancy Age & Remarried (second or else) & $3.3 \%$ \\
& First & $52.9 \%$ \\
& Second & $32.2 \%$ \\
Miscarriage Experience & Other & $14.9 \%$ \\
Depression & $1^{\text {st }}$ Trisemester & $13.2 \%$ \\
& $2^{\text {nd }}$ Trisemester & $43 \%$ \\
& $3^{\text {rd }}$ Trisemester & $43.8 \%$ \\
& Yes & $6.6 \%$ \\
& No & $93.4 \%$ \\
& No Depression & $67.8 \%$ \\
& Depression & $32.2 \%$ \\
\hline & & \\
& &
\end{tabular}

Table 2. Correlation Test Results

\begin{tabular}{clc}
\hline & & Depression \\
\hline \multirow{2}{*}{ Mindfulness } & -0.043 \\
& Describing & $-0.292^{* *}$ \\
& Acting with Awareness & $-0.514^{* *}$ \\
& Non-Judging & $-0.445^{* *}$ \\
& Non-Reactivity & 0.007 \\
& Self-Compassion & $-0.553^{* *}$ \\
\hline$* *$ Correlation is significant at the 0.01 level (2-tailed).
\end{tabular}

Table 3. Multiple Regression Test Results

\begin{tabular}{cccc}
\hline Model & R-Square & F & Sig. (p) \\
\hline 1 & 0.424 & 21.35 & $0.000^{\mathrm{b}}$ \\
\hline
\end{tabular}

Table 4. B-Values \& Sig

\begin{tabular}{clccc}
\hline Model & & Sig. & B & Beta \\
\hline \multirow{2}{*}{1} & (Constant) & 0.000 & 34.771 & \\
& Describing & 0.009 & -0.186 & -0.203 \\
& Self-Compassion & 0.000 & -0.118 & -0.340 \\
\hline
\end{tabular}

\subsection{Discussions}

This study conducted a study to know the relationship and the role of mindfulness and selfcompassion toward depression in pregnant women. Based on the results obtained, it showed that selfcompassion had a significant relationship with depression, whereas for mindfulness, only the dimensions of describing, acting with awareness, and non-judging were found to have a significant relationship with depression. Moreover, multiple regression analysis was conducted to find the role of mindfulness and selfcompassion towards depression. The results showed that mindfulness and self-compassion had a significant role toward depression on pregnant women. The research conducted by Pereira, Xavier, Bento, and 
Macedo [27], also found that mindfulness and self-compassion were protective factors towards antenatal depressive symptoms.

Based on literature study conducted by Matvienko-Sikar, Lee, Murphy and Murphy [27], it was found that various mindfulness interventions performed on pregnant women could reduce depression on pregnant women. Decreased levels of depression was caused by an increase in the level of mindfulness possessed by pregnant women. This increased level of mindfulness could be occured by using mindfulness intervention. Mindfulness interventions conducted by Goodman et al [18] also include self-compassion in the intervention process, with the consideration that self-compassion was an essential component of mindfulnessrelated interventions and was associated significantly with decreased anxiety, depression and overall psychological health. The results showed that participants who were able to complete the intervention had significant improvement in reducing the severity of depression they experienced. This reduction in the severity of the depression coincided with a significant increase in mindfulness and self-compassion in participants.

The results of this study indicated that only describing dimensions had a significant contribution toward depression on pregnant women. This was in line with previous research that some dimensions in mindfulness, ie non-judging of experience, acting with awareness, observing and describing, as well as selfcompassion have a significant relationship to antenatal depressive symptoms [26]. In previous studies, conducted on undergraduate, it is also known that describing dimensions are a strong predictor of depression compared to other dimensions of mindfulness [28].

The non-reactivity dimension was found to have no association with depression in pregnant women in this study. The result of non-reactivity to inner experience that has no relationship to depression can be influenced by culture. Indonesia has a collectivistic culture so that individuals tend to try to adapt to how their environment behaves [29]. If a person is not reactive to his thoughts or feelings and does not seek to evaluate himself, then this is opposed to the collectivist culture prevailing in Indonesia [29].

In addition, observing dimensions were also found to have no association with depression in pregnant women in this study. Baer et al [30] also found that observing may change depending on whether the subject is meditating regularly or not. In observing, one is aware of every conscious stimulus such as, sensations and emotions. One's attention becomes focused on them. This may be associated with negative clinical and non-clinical emotions, psychological disorders and other maladaptive matters [30]. However, observing can be a positive thing in mindfulness training [30]. In mindfulness training, one is taught to focus with internal stimuli and teaches participants to accept, non-judging, and non-reactive even though the stimulus is uncomfortable for them.

\section{CONCLUSION}

The dimension of describing the mindfulness and self-compassion has a role to decrease depression in pregnant women. This suggests that pregnant women in this study still need to be helped understand mindfulness as a whole. A follow-up study is suggested to be done regarding mindfulness-based interventions to assist pregnant women in overcoming depression, with attention to handling involving all existing facet mindfulness, so expect the future of pregnant women to improve their psychological health condition and reduce their depression.

\section{REFERENCES}

[1] Krucik, G., "What Body Changes Can You Expect During Pregnancy?" Accessed 30 November 2017, http://www.healthline.com/health/pregnancy/bodily-changesduring\#HormonalChanges1, 2012.

[2] Duncan, L.G., Cohn, M. A., Chao, M. T., Cook, J. G., Riccobono, J., \& Bardacke, N., "Benefits of Preparing for Childbirth with Mindfulness Training: a Randomized Controlled Trial with Active Comparison”, BMC Pregnancy and Childbrith, 17, 140. DOI 10.1186/s12884-017-1319-3, 2017.

[3] Aktas, S., \& Calik, K. Y., "Factors Affecting Depression during Pregnancy and the Correlation between Social Support and Pregnancy Depression" Iranian Red Crescent Medical Journal, 17(9), e16640. DOI: 10.5812/ircmj.16640, 2015.

[4] Gavin, N. I., Gaynes, B. N., Lohr, K. N., Meltzer-Brody, S., Gartlehner, G., \& Swinson, T., "Perinatal Depression: A Systematic Review of Prevalence and Incidence", Obstetrics \& Gynecology, 106(5), 1071-1083. DOI: 10.1097/01.AOG.0000183597.31630.db, 2005.

[5] Bennet, H. A., Einarson, A., Taddio, A., Koren, G., \& Einarson T. R., "Prevalence of Depression during Pregnancy: Systematic Review", Obstetrics \& Gynecology, 103, (4), 698-709. DOI: 10.1097/01.AOG.0000116689.75396.5f, 2004.

[6] Roomruangwong, C., \& Epperson, C. N., "Perinatal Depression in Asian Women: Prevalence, Associated Factors, and Cultural Aspects”, Asian Biomedicine, 5(2), 179-193. DOI: 10.5372/1905-7415.0502.024, 2011. 
[7] Fauzy, R., \& Fourianalistyawati, E., "The Relationship between Depression and Quality of Life in High-risk Pregnant (in Bahasa)", Jurnal Psikogenesis, 4(2), 206-214, 2016.

[8] Handayani, F. P., \& Fourianalistyawati, E., "The Relationship between Depression and Spiritual Well-Being in High-Risk Pregnant (in Bahasa)", Thesis: Universitas YARSI, 2015.

[9] Deave, T., Heron, J., Evans, J., \& Emond, A., "The Impact of Maternal Depression in Pregnancy on Early Child Development", BJOG: An International Journal of Obstetrics \&Gynaecology, 115(8), 1043-1051. DOI: 10.1111/j.1471-0528.2008.01752.x, 2008.

[10] Alder, J., Fink, N., Bitzer, J., Hosli, I., \& Holzgreve, W., "Depression and Anxiety during Pregnancy: A Risk Factor for Obstetric, Fetal and Neonatal Outcome? A Critical Review of the Literature", The Journal of Maternal-Fetal and Neonatal Medicine, 20(3), 189-209. DOI: 10.1080/14767050701209560, 2007.

[11] Dunkel Schetter, C., \& Tanner, L., "Anxiety, Depression and Stress in Pregnancy: Implications for Mothers, Children, Research, and Practice", Current Opinion in Psychiatry, 25(2), 141-148, 2015.

[12] Osborne, S., \& O'Keane, V., "Management of Depression during Pregnancy", Progress in Neurology and Psychiatry, 13(2), 6-11. DOI: 10.1002/pnp.113, 2009.

[13] Badker, R., \& Misri, S., "Mindfulness-based Therapy in the Perinatal Period: A review of the Literature", $B C$ Medical Journal, 59(1), 18-21, 2017.

[14] Kabat-Zinn, J., "Mindfulness-based Interventions in Context: Past, Present, and Future", Clinical Psychology: Science and Practice, 10(2), 144-156. DOI: 10.1093/clipsy.bpg016, 2003.

[15] Baer, R. A., Lykins, E. L. B., \& Peters, J. R., "Mindfulness and Self-Compassion as Predictors of Psychological Wellbeing in Long-Term Meditators and Matched Nonmeditators", The Journal of Positive Psychology: Dedicated to furthering research and promoting good practice, 7(3), 230-238. DOI: 10.1080/17439760.2012.674548, 2012.

[16] Yazdanimehr, R., Omidi, A., Sadat, Z., \& Akbari, H., "The Effect of Mindfulness-Integrated Cognitive Behavior Therapy on Depression and Anxiety among Pregnant Women: A Randomized Clinical Trial", Journal of Caring Sciences, 5(3), 195-204. DOI: 10.15171/jcs.2016.021, 2016.

[17] Tomfohr-Madsen, L. M., Campbell, T. S., Giesbrecht, G. F., Letourneau, N. L., Carlson, L. E., Madsen, J. W., \& Dimidjian, S., "Mindfulness-based cognitive therapy for psychological distress in pregnancy: study protocol for a randomized controlled trial" Trials, 17, 498. http://doi.org/10.1186/s13063-016-1601-0, 2016.

[18] Goodman, J. H., Guarino, A., Chenausky, K., Klein, L., Prager, J., Petersen, R., Freeman, M., "CALM Pregnancy: Results of a Pilot Study of Mindfulness-Based Cognitive Therapy for Perinatal Anxiety", Archives of Women's Mental Health, 17(5), 373-387. http://doi.org/10.1007/s00737-013-0402-7, 2014.

[19] Viesten, C., \& Astin, J., "Effects of a Mindfulness-Based Intervention during Pregnancy on Prenatal Stress and Mood: Results of a Pilot Study", Archives of Women's Mental Health, 11, 67-74. DOI: 10.1007/s00737-008-0214-3, 2007.

[20] Baer, R. A., Smith, G. T., Hopkins, J., Krietemeyer, J., \& Toney, L., "Using self-Report Assessment Methods to Explore Facets of Mindfulness", Assessment, 13(27), 27-45. DOI: 10.1177/1073191105283504, 2006.

[21] Felder, J. N., Lemon, E., Shea, K., Kripke, K., \& Dimidjian, S., "Role of Self-Compassion in Psychological WellBeing among Perinatal Women”, Archives of Women's Mental Health, 19(4), 687-690. DOI: 10.1007/s00737-0160628-2, 2015

[22] Neff, K. D., "Self-compassion: An Alternative Conceptualization of a Healthy Attitude toward Oneself" Self and Identity, 2, 85-102, 2003.

[23] Neff, K. D., "The Development and Validation of a Scale to Measure Selfcompassion", Self and Identity, 2, 223250. DOI: $10.1080 / 15298860390209035,2003 \mathrm{a}$.

[24] Mohamadirizi, S., \& Kordi, M., "The Relationship between Multi-Dimensional Self-Compassion and FetalMaternal Attachment in Prenatal Period in Reffered Women to Mashhad Health Center", Journal of Education and Health Promotion, 5:21, 2016.

[25] Cox, J. L., Holden, J. M., \& Sagovsky, R., "Detection of Postnatal Depression: Development of the 10-Item Edinburgh Postnatal Depression Scale”, British Journal of Psychiatry, 150, 782-786, 1987.

[26] Pereira, A. T., Xavier, S., Bento, E., Azevedo, J., Marques, M., Soares, M. J., Macedo, A. F., "Mindfulness, SelfCompassion and Depressive Symptoms in Pregnant Women", $24^{\text {rd }}$ European Congress of Psychiatry, 2016. Europe: European Psychiatry 33S. DOI: http://dx.doi.org/10.1016/j.eurpsy.2016.01.1517, 2016.

[27] Matvienko-Sikar, K., Lee, L., Murphy, G., \& Murphy, L., "The Effects of Mindfulness Interventions on Prenatal Well-Being: A Systematic Review", Psychology \& Health. DOI: 10.1080/08870446.2016.1220557, 2016.

[28] Soysam, C. K., \& Wilcomb, C. J., "Mindfulness, Sell-Compassion, Self-Efficacy, and Gender as Predictors of Depression, Anxiety, Stress, and Well-Being", Mindfulness. DOI: 10.1007/s12671-013-0247-1, 2013.

[29] Sanoveriana, A. S. N., \& Fourianalistyawati, E., "Work-Family Balance, Trait Mindfulness and Psychological Well-Being in Middle-Aged Working Parents", Universitas Indonesia Psychology Symposium for Undergraduate Research (UIPSUR 2016), Universitas Indonesia, 2016. Jakarta, Indonesia: Universitas Indonesia, 2016.

[30] Baer, R. A., Smith, G. T., Lykins, E., Button, D., Krietemeyer, J., Sauer, S., Walsh, E., Duggan, D., \& Williams, M. G., "Construct Validity of the Five Facet Mindfulness Questionnaire in Meditating and non Meditating Samples", Assessment, 15, 329-342. DOI: 10.1177/1073191107313003, 2008. 